\title{
Distortion Performance of Convolutionally Coded Underwater Acoustic Networks
}

\author{
Andrej Stefanov
}

\begin{abstract}
The paper studies the impact of point-to-point convolutional channel coding on the sustainable number of hops through the network for a maximum allowed route distortion requirement. The network is modeled by a uniform distribution of nodes over a finite area. The node-to-node channel is described by the Ricean fading model, while the interference from other nodes in the network is modeled as Gaussian. Numerical examples are presented to illustrate the results of the analysis.
\end{abstract}

Keywords — underwater acoustic networks, fading.

\section{Introduction}

There have been significant recent research efforts focused on the analysis and design of underwater networks [1-4], including medium access control [5-8], and routing protocols [9-13]. The study of underwater acoustic networks needs to take into consideration a number of design aspects that arise due to the underwater transmission medium. This includes the limitations of the operational bandwidth and path loss that depends on the signal frequency, in addition to the distance between the transmitter and the receiver. Due to these challenges posed by underwater communications, the selection of the operational frequency requires careful consideration.

The paper studies an underwater network of nodes that are mounted at the bottom, hence the network is modeled by a two dimensional model. The node distribution is assumed to be uniform over a finite area. Multihop routing among nearest neighbor nodes is considered. The focus is on the impact of point-to-point convolutional channel coding on the distortion performance of the multihop network; given by the sustainable number of hops in the network for a target route distortion requirement in the presence of interference from other nodes in the network.

The paper is organized as follows. The underwater acoustic propagation model and the set-up of the network are discussed in Sections II and III, respectively. The impact of point-to-point convolutional coding on the sustainable number of hops in the network for a target route distortion requirement is investigated in Section III. Numerical examples are illustrated in Section IV. The paper concludes with Section V.

Andrej Stefanov

IBU Skopje

Macedonia

\section{Underwater Acoustic Propagation}

Underwater acoustic communication is affected by a path loss that depends on the distance between the transmitter and the receiver, and the frequency of the transmitted signal. The path loss increases with distance between the transmitter and the receiver and with the transmitted signal frequency thereby imposing a limit on the transmission bandwidth.

Specifically, underwater acoustic propagation experiences a path loss, which at distance $d$ between the transmitter and the receiver, and for a signal transmitted on frequency $f$, is

$$
A(d, f)=A_{0} d^{\kappa} a(f)^{d}
$$

where $A_{0}$ is a unit-normalizing constant that incorporates fixed losses, $a(f)$ is the absorption coefficient and $\kappa$ is the spreading factor. Usually $1 \leq \kappa \leq 2$. It is commonly assumed that for practical spreading, $\kappa=1.5$. Using Thorp's formula which gives $a(f)$ in $\mathrm{dB} / \mathrm{km}$ for $f$ in $\mathrm{kHz}$, the absorption coefficient is

$$
10 \log a(f)=\frac{0.11 f^{2}}{1+f^{2}}+\frac{44 f^{2}}{4100+f^{2}}+\frac{2.75 f^{2}}{10^{4}}+0.003
$$

This formula is typically valid for frequencies above several hundred $\mathrm{Hz}$, the common range for most systems.

The ocean ambient noise is modeled as composed of turbulence, shipping, waves and thermal noise, described by Gaussian statistics and a continuous power spectral densities (p.s.d.). The respective noise components have formulae that give their p.s.d.'s in $\mathrm{dB}$ re $\mu \mathrm{Pa}$ per $\mathrm{Hz}$ as a function of frequency in $\mathrm{kHz}$ [14]:

$$
\begin{gathered}
10 \log N_{\mathrm{t}}(f)=17-30 \log f \\
10 \log N_{\mathrm{s}}(f)=40+20(s-0.5)+26 \log f \\
-60 \log (f+0.03) \\
10 \log N_{\mathrm{w}}(f)=50+7.5 \sqrt{w}+20 \log f \\
-40 \log (f+0.4) \\
10 \log N_{\mathrm{th}}(f)=-15+20 \log f
\end{gathered}
$$

where $s$ is the shipping activity factor and $w$ is the wind speed in $\mathrm{m} / \mathrm{s}$. The total noise p.s.d. is

$$
N(f)=N_{\mathrm{t}}(f)+N_{\mathrm{s}}(f)+N_{\mathrm{w}}(f)+N_{\mathrm{th}}(f) .
$$




\section{Network Setup}

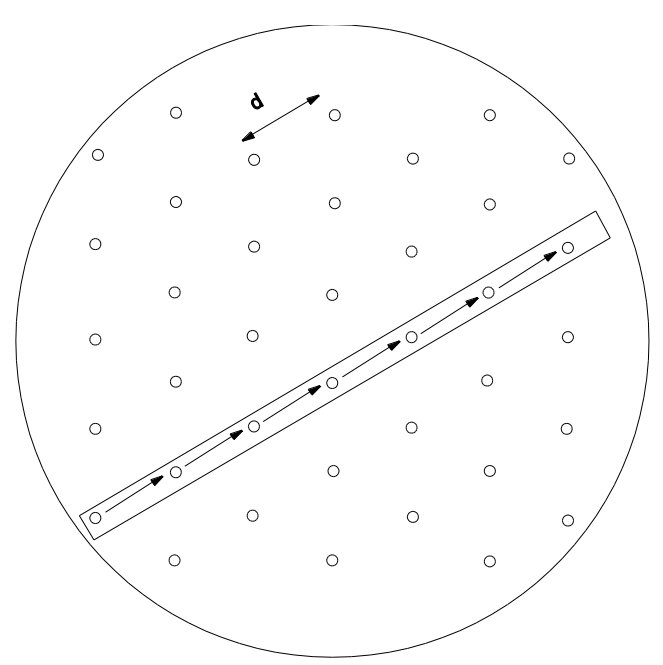

Fig. 1. The setup of the uniform network.

\section{A. Network Topology}

The topology is based on a two dimensional network model of nodes that are bottom mounted and provide coverage over a finite area [4]. The node distribution is assumed to be uniform as shown in Figure 1. The network density is

$$
\rho=\frac{N}{\mathcal{A}}
$$

where $N$ is the number of nodes in the network and $\mathcal{A}$ is the network area. The distance between neighboring nodes is

$$
d=\frac{c}{\sqrt{\rho}}
$$

where $c$ is a constant that depends on the placement of the nodes (grid pattern). We have $c=1$.

Node-to-node multihop routing is considered. This is a strategy that is energy efficient in the context of underwater acoustic networks. As such, it is appealing for battery powered nodes. The maximum number of hops in the network is $n_{h}^{\max }=(2 / \sqrt{\pi}) \sqrt{N}$. The average number of hops for a multihop route is designated by $\bar{n}_{h}$. We have $\bar{n}_{h}=\sqrt{N / \pi}[15]$.

\section{B. Coded Multihop Transmission}

In the case of point-to-point convolutional channel coding with BPSK modulated communication, the union upper bound on the bit error probability (BEP) is [16]

$$
p_{b}<\sum_{d=d_{\min }} c_{d} p_{d}
$$

where $c_{d}$ is the information error weight for error events of distance $d, p_{d}$ is the pairwise error probability and $d_{\text {min }}$ is the code minimum distance.

\section{1) Interference Model:}

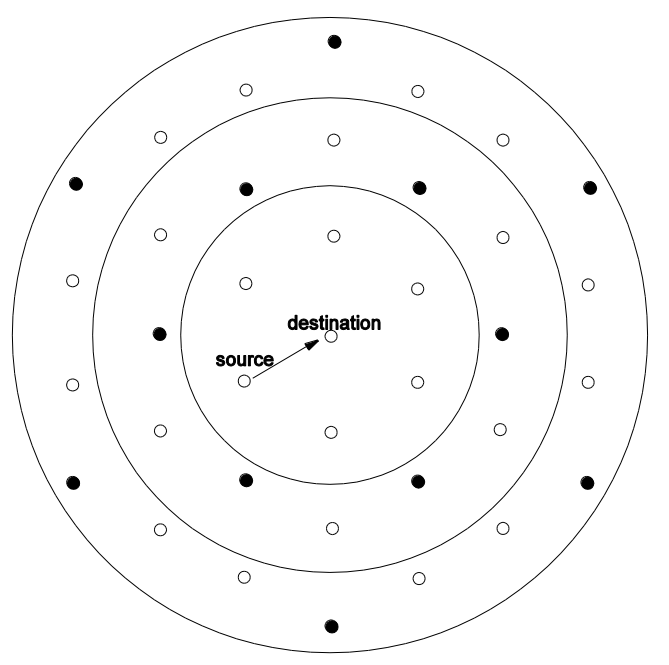

Fig. 2. The interference model.

Consider a source to a destination transmission as illustrated in Figure 2. The considered interference model is based on a protocol constraint [4], where nodes which distance to the destination is the same as the source node distance to the destination are not permitted to transmit on the same frequency and in the same time slot as the source during its transmission.

As it is assumed that all nodes transmit with a constant p.s.d. $S$, the total interference from the nodes in the first and second tier is

$$
I(f) \approx \frac{c_{1} S}{A(2 d, f)}+\frac{c_{2} S}{A(3 d, f)}
$$

where $c_{1} \leq 12$ and $c_{2} \leq 18$ represent constants that indicate how many interfering nodes are in tiers 1 and 2 , respectively. In the Figure 2 example, $c_{1}=c_{2}=6$. Since there are multiple nodes that contribute to the interference in the network, it is assumed that the interference is Gaussian with p.s.d. $I(f)$.

Assuming perfect channel state information at the receiver and flat Ricean fading for the node-to-node channel [16, 17], the BEP is

$$
\begin{aligned}
p_{b} & <\sum_{d=d_{\min }} c_{d}\left(\frac{1+\mathcal{K}}{1+\mathcal{K}+R \gamma(d, f)}\right)^{d} \\
& \times \exp \left(-d R \frac{\mathcal{K} \gamma(d, f)}{1+\mathcal{K}+R \gamma(d, f)}\right)
\end{aligned}
$$

where $\gamma$ is the signal to interference plus noise ratio (SINR) and $\mathcal{K}$ is the Ricean fading parameter. It is assumed that the attenuation, noise and interference are constant over the operational bandwidth $B$, so that for a transmit power level $P$, the SINR evaluated at the operating frequency $f_{o}(d)$ is

$$
\gamma\left(d, f_{o}\right)=\frac{P}{A\left(d, f_{o}\right)\left(N\left(d, f_{o}\right)+I\left(d, f_{o}\right)\right) B} .
$$




\section{Distortion Performance}

The distortion performance is considered for an independent identically distributed (i.i.d.) Gaussian source. For single description coding, the distortion is $D=\sigma^{2} 2^{-2 R}$ [18], where $\sigma^{2}$ is the variance of the i.i.d. Gaussian random variables and $R$ is the bit rate of an optimal source coder. The sustainable number of hops is [19]

$$
n_{\mathrm{sh}}=\frac{1}{L} \cdot \frac{1}{p_{b}} \cdot \frac{1}{\sigma^{2}} \cdot\left(D_{\text {route }}^{\max }-D\right)
$$

where $D_{\text {route }}^{\max }$ is the maximum allowed route distortion.

\section{Numerical Results}

The aim of the numerical examples is to examine the impact of point-to-point convolutional coding on the network connectivity [20], given by the sustainable number of hops, for a maximum allowed route distortion requirement. The maximum allowed route distortion is required to be within $10 \%$ of the optimum achievable distortion, in other words, $\left(D_{\text {route }}^{\max }-D\right) \times 100 \%=10 \%$. The rate of the source coder is $R=4$ bits per description. Independent Ricean fading for each node-to-node channel with $K=10$ is assumed. The circular network area is $\mathcal{A}=1000 \mathrm{~km}^{2}$. Fixed losses are neglected [4]. The frame size is $L=100$ bits, and the bandwidth is $B=4 \mathrm{kHz}$. The spreading factor is $\kappa=1.5$, the shipping activity factor is $s=0.5$, and unless otherwise indicated, the wind speed is $w=0$.

Figure 3 presents the sustainable number of hops when there is interference in the network, as illustrated in Figure 2. The transmit power is $P=130 \mathrm{~dB}$ re $\mu \mathrm{Pa}$. As illustrated in Figure 3, uncoded BPSK modulated communication with demodulate and forward relaying cannot provide connectivity in the network regardless of how many nodes are present in the network. Point-to-point convolutional channel coding with the rate $R=1 / 2,(133,171)$ code, on the other hand, provides full connectivity in the network.

Figure 4 provides a comparison among the sustainable number of hops, when there is interference in the network, between convolutional channel coding with the rate $R=1 / 2,(133,171)$ code and the stop and wait ARQ protocol [19] with different number of retransmissions, J. The transmit power is $P=124 \mathrm{~dB}$ re $\mu \mathrm{Pa}$. As illustrated in Figure 4, in the case of the stop and wait ARQ protocol, the network connectivity improves with the increase of the number of possible retransmissions. When $\mathbf{J}=1$, the stop and wait ARQ protocol cannot provide full connectivity regardless of how many nodes are present in the network. It is however possible to maintain routes with an average number of hops when the number of nodes in the network is $300 \lesssim N \lesssim 900$. Of course, when $N \lesssim 300$ and $N \gtrsim 900$ even routes with an average number of hops cannot be supported by the stop and wait ARQ protocol with a single retransmission. When the number of retransmissions is increased to two, that is, when $J=2$, the performance of the stop and wait ARQ protocol improves. In this case, the stop and wait ARQ protocol can provide full connectivity unless the number of nodes in the network is $N \lesssim 50$, in which case the network becomes coverage limited. This means that the nodes are too far apart to ensure connectivity in the network for the given transmit power. In comparison, multihop transmission with point-to-point convolutional channel coding provides full connectivity in the network.

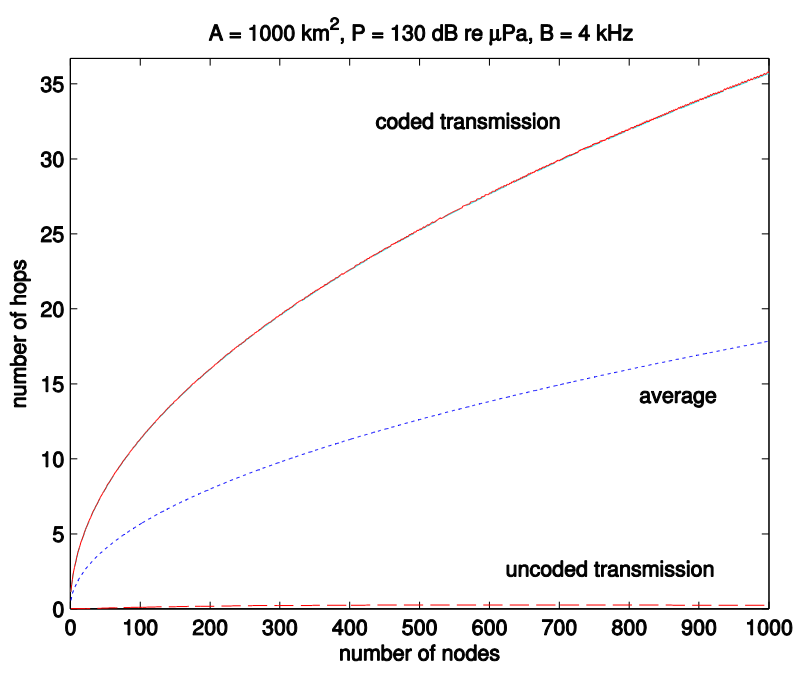

Fig. 3. Sustainable number of hops for $\boldsymbol{R}=\mathbf{4}$ bits per description and $\left(D_{\text {route }}^{\max }-\boldsymbol{D}\right) \times \mathbf{1 0 0} \%=\mathbf{1 0} \%$. Uncoded BPSK vs. rate $1 / 2,(133,171)$ convolutional coding.

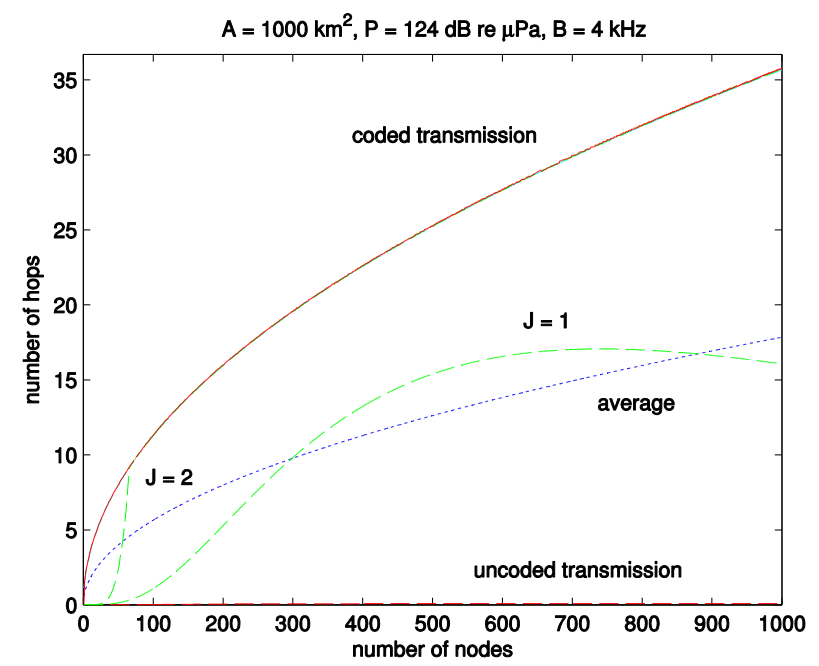

Fig. 4. Sustainable number of hops for $\boldsymbol{R}=\mathbf{4}$ bits per description and $\left(D_{\text {route }}^{\max }-D\right) \times \mathbf{1 0 0} \%=\mathbf{1 0} \%$ for rate $1 / 2,(133,171)$ convolutional coding and stop and wait ARQ with different number of retransmissions. 
Figure 5 illustrates the sustainable number of hops for different wind speeds when there is interference in the network for point-to-point convolutional channel coding with the rate $R=1 / 2,(133,171)$ code. The transmit power is $P=104 \mathrm{~dB}$ re $\mu \mathrm{Pa}$. Even at this reduced transmit power level, under calm conditions, that is $w=0 \mathrm{~m} / \mathrm{s}$, the network provides full connectivity unless the number of nodes in the network is $N \lesssim 50$. However, if the wind speed increases to $w=2 \mathrm{~m} / \mathrm{s}$, and $w=4 \mathrm{~m} / \mathrm{s}$, the network connectivity is significantly affected as the network becomes coverage limited when the number of nodes in the network is $N \lesssim 400$, and $N \lesssim 900$, respectively.

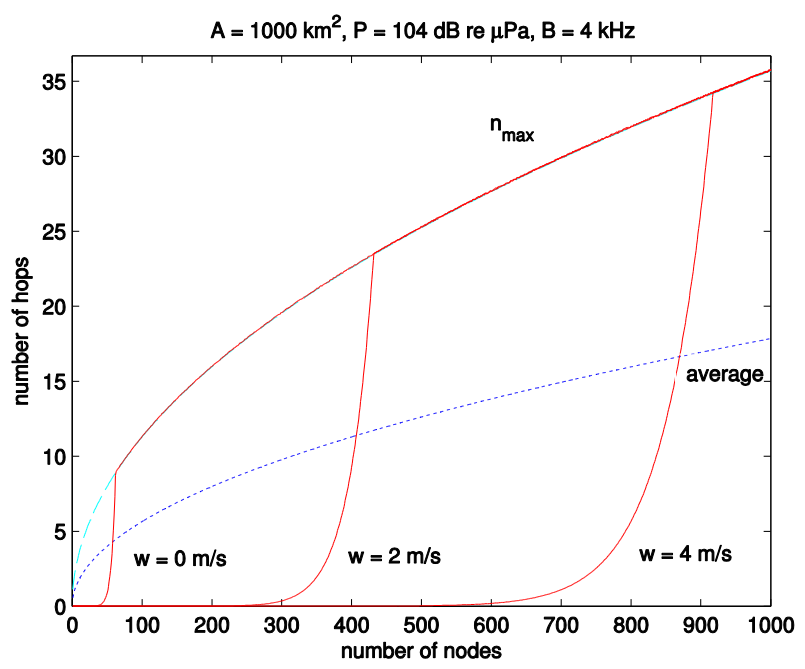

Fig. 5. Sustainable number of hops for $\boldsymbol{R}=\mathbf{4}$ bits per description and $\left(D_{\text {route }}^{\max }-D\right) \times \mathbf{1 0 0} \%=\mathbf{1 0} \%$ for different wind speeds. Rate $\mathbf{1} / \mathbf{2},(\mathbf{1 3 3}, \mathbf{1 7 1})$ convolutional coding.

\section{v. Conclusions}

The paper considered the distortion performance of convolutionally coded underwater acoustic multihop networks in the presence of interference. In particular, the distortion performance was considered for an independent identically distributed (i.i.d.) Gaussian source and single description coding. It was found that point-to-point convolutional channel coding significantly improves the network connectivity in comparison to uncoded BPSK modulated communication with demodulate and forward relaying, and the stop and wait ARQ protocol. The robustness with respect to increased wind speeds was also investigated. Numerical examples, based on the union upper bound on the BEP of convolutional channel codes, illustrating the sustainable number of hops in the network were presented to illustrate the results of the analysis.

\section{References}

[1] Special Issue on Underwater Wireless Communications and Networks, IEEE Journal Sel. Areas Commun., vol. 26, Dec. 2008.

[2] Special Issue on Underwater Networks, Ad Hoc Networks, vol. 7, Elsevier, Jun. 2009.

[3] Special Issue on Underwater Sensor Nodes and Underwater Sensor Networks, Sensors, MDPI Publishing, 2011-2012.

[4] A. Stefanov and M. Stojanovic, "Design and Performance Analysis of Underwater Acoustic Networks," IEEE Journal Sel. Areas Commun., vol. 29, pp. 2012-2021, Dec. 2011.

[5] A. A. Syed, W. Ye, and J. Heidemann, "Comparison and Evaluation of the T-Lohi MAC for Underwater Acoustic Sensor Networks," IEEE Journal Sel. Areas Commun., vol. 26, pp. 1731-1743, Dec. 2008.

[6] Y. Xu, J. Mao, S. Chen, J. Yu, Y. Gu, and R. Yu, "LTM-MAC: A Location-Based TDMA MAC Protocol for Mobile Underwater Networks," in Proc. of MTS/IEEE OCEANS, Shanghai, China, Apr. 2016.

[7] Y.-K. Chen, F. Ji, Q. Guan, F. Chen, and H. Yu, "A New MAC Based on RTT Prediction for Underwater Acoustic Networks," in Proc. of the $11^{\text {th }}$ ACM International Conference on Underwater Networks and Systems, Shanghai, China, Oct. 2016.

[8] K. Chen, M. Ma, E. Cheng, F. Yuan, and W. Su, "A Survey on MAC Protocols for Undewater Wireless Sensor Networks," IEEE Communications Surveys and Tutorials, vol. 16, pp. 1433-1447, Mar. 2014.

[9] P. Nicopolitidis, G. I. Papadimitrou, and A. S. Pomportsis, "Adaptive Data Broadcasting in Underwater Wireless Networks," IEEE Journal of Oceanic Engineering, vol. 35, pp. 623-634, Jul. 2010.

[10] A. K. Mohapatra, N. Gautam, and R. L. Gibson, "Combined Routing and Node Replacement in Energy Efficient Underwater Sensor Networks for Seismic Monitoring," IEEE Journal of Oceanic Engineering, vol. 38, pp. 80-90, Jan. 2013.

[11] N. Javaid et al., "Delay Sensitive Routing Schemes for Underwater Acoustic Sensor Networks," International Journal of Distributed Sensor Networks, vol. 11, 3:532676, Mar. 2015.

[12] H.-H. Cho, C.-Y. Chen, T.-K. Shih, H.-C. Chao, "Survey on Underwater Delay/Disruption Tolerant Wireless Sensor Network Routing," IET Wireless Sensor Systems, vol. 4, pp. 112-121, Sep. 2014.

[13] N. Li, J.-F. Martinez, J. M. M. Chaus, and M. Eckert, "A Survey on Underwater Acoustic Sensor Network Routing Protocols," Sensors, vol. 16, MDPI Publishing, Mar. 2016.

[14] L. Brekhovskikh and Y. Lysanov, "Fundamentals of Ocean Acoustics," Springer, 1982.

[15] O. Tonguz and G. Ferrari, "Ad Hoc Wireless Networks: A Communication-Theoretic Perspective," Wiley, 2006.

[16] S. Benedetto and E. Biglieri, "Principles of Digital Transmission with Wireless Applications," Kluwer/Plenum, 1999.

[17] P. Qarabagi and M. Stojanovic, "Statistical Characterization and Computationally Efficient Modeling of a Class of UnderwaterAcoustic Communication Channels," IEEE Journal of Oceanic Engineering, vol. 38, pp. 701-717, Oct. 2013.

[18] T. M. Cover and J. A. Thomas, "Elements of Information Theory," Wiley, 1991.

[19] A. Stefanov, "Distortion Performance of Underwater Acoustic Sensor Networks," International Journal of Distributed Sensor Networks, Vol. 11, 10:352569, Oct. 2015.

[20] A. Stefanov, "Performance of Convolutionally Coded Underwater Acoustic Ad-Hoc Networks," Proceedings of the International Conference on Advances in Information Processing and Communication Technology, pp. 115-118, Rome, Italy, Dec. 2015. 